Review

\title{
A Review of Ten Years of the Study for Monitoring Antimicrobial Resistance Trends (SMART) from 2002 to 2011
}

\author{
Ian Morrissey $^{1}$, , Meredith Hackel ${ }^{2}$, Robert Badal ${ }^{2}$, Sam Bouchillon ${ }^{2}$, \\ Stephen Hawser ${ }^{1}$ and Douglas Biedenbach ${ }^{2}$ \\ 1 IHMA Europe Sàrl, 9A Route de la Corniche, Epalinges 1066, Switzerland \\ 2 International Health Management Associates, Inc; Schaumburg, IL 60173, IL, USA \\ * Author to whom correspondence should be addressed; E-Mail: imorrissey@ihmainc.com; \\ Tel: +44-(0)-1279-724-929.
}

Received: 29 September 2013; in revised form: 26 October 2013 / Accepted: 28 October 2013 / Published: 1 November 2013

\begin{abstract}
Surveillance of antimicrobial agent resistance provides important information to guide microbiologists and infectious disease specialists understanding of the control and the spread of resistance mechanisms within the local environment. Continued monitoring of antimicrobial resistance patterns in the community and in local hospital environments is essential to guide effective empiric therapy. The Study for Monitoring Antimicrobial Resistance Trends (SMART) has monitored the in vitro susceptibility patterns of clinical Gram-negative bacilli to antimicrobial agents collected worldwide from intra-abdominal infections since 2002 and urinary tract infections since 2009. Resistance trends, with a particular focus on carbapenem resistance and the rate of extended-spectrum $\beta$-lactamases (ESBLs), were analyzed. Isolates from intra-abdominal infections $(\mathrm{n}=92,086)$ and urinary-tract infections $(n=24,705)$ were collected and tested using Clinical and Laboratory Standards Institute methods. This review presents carbapenem susceptibility and ESBL rates over ten years of SMART study analysis, including key publications during this period. The SMART study has proved to be a valuable resource in determining pathogen prevalence and antibiotic susceptibility over the last ten years and continues to provide evidence for regulatory susceptibility breakpoints and clinical decision making.
\end{abstract}

Keywords: carbapenems; surveillance; Gram-negative; extended-spectrum $\beta$-lactamase 


\section{Introduction}

Surveillance of antimicrobial resistance in frequently isolated human pathogens can help to develop rational therapies [1]. Hospital studies can guide local antimicrobial agent use or larger studies can collect isolates for resistance testing at a central microbiology laboratory. Data collected from such studies can be used to guide microbiologists and infection disease specialists in the control and containment of resistant pathogens. Although antibiotics have been in use since the 1940s, coordinated multicentre surveillance studies were not considered until the early 1990s. One of the earliest of these surveillance studies was the Alexander project which began in 1992 and focused on respiratory tract infections [2]. Since then, other global studies have provided additional scientific data with regards to antimicrobial resistance. These include studies such as EARS-net, PROTEKT, SENTRY and TEST which are discussed elsewhere [1].

The Study for Monitoring Antimicrobial Resistance Trends (SMART) is an ongoing global surveillance study. This investigation has determined the in vitro resistance of clinical Gram-negative bacilli to antimicrobial agents from intra-abdominal infections (IAIs) since 2002 and was extended further to provide antimicrobial resistance data and pathogen prevalence from urinary tract infections (UTIs) in 2009 worldwide, with a particular focus on carbapenems and extended-spectrum $\beta$-lactamases (ESBLs). The two main objectives of the SMART study were to monitor the in vitro resistance in Gram-negative bacilli to antimicrobials IAIs and UTIs and identify changes in resistance patterns in community- or hospital-acquired organisms, including those that produce ESBLs. This review presents some newly analysed data for carbapenem resistance and ESBLs over ten years of the SMART study and discusses key publications from the study during this period.

\section{Materials and Methods}

\subsection{Isolate Collection and Sites}

Hospital laboratories from multinational locations were invited to collect up to 100 consecutive Gram-negative bacilli from patients with intra-abdominal infections and 50 Gram-negative isolates from urinary-tract infections each year. The collection only includes unique bacterial isolates and excludes duplicate isolates. Isolates were differentiated on the duration of hospitalization $(<48 \mathrm{~h}$ or $\geq 48 \mathrm{~h}$ ) at time of isolate recovery. Isolates recovered at $<48 \mathrm{~h}$ of hospitalization were considered community-associated and those recovered at $\geq 48 \mathrm{~h}$ of hospitalization were considered to be hospital-associated. Isolates were identified and confirmed as Gram-negative bacilli and then shipped to a central microbiology laboratory (International Health Management Associates, Inc, Schaumburg, IL, USA). These isolates were re-identified at the central laboratory using biochemical methods as appropriate. In total 282 hospital sites have participated in the SMART program since 2002. The number of participating sites has grown from 40 in 2002 to 179 in 2011 . The location of these laboratories for 2011 is shown in Figure 1. There were 165 sites that participated in three or more years; 71 that participated for five or more years; and 13 sites have participated in all 10 years. 
Figure 1. Map indicating SMART sites as participating in 2011.

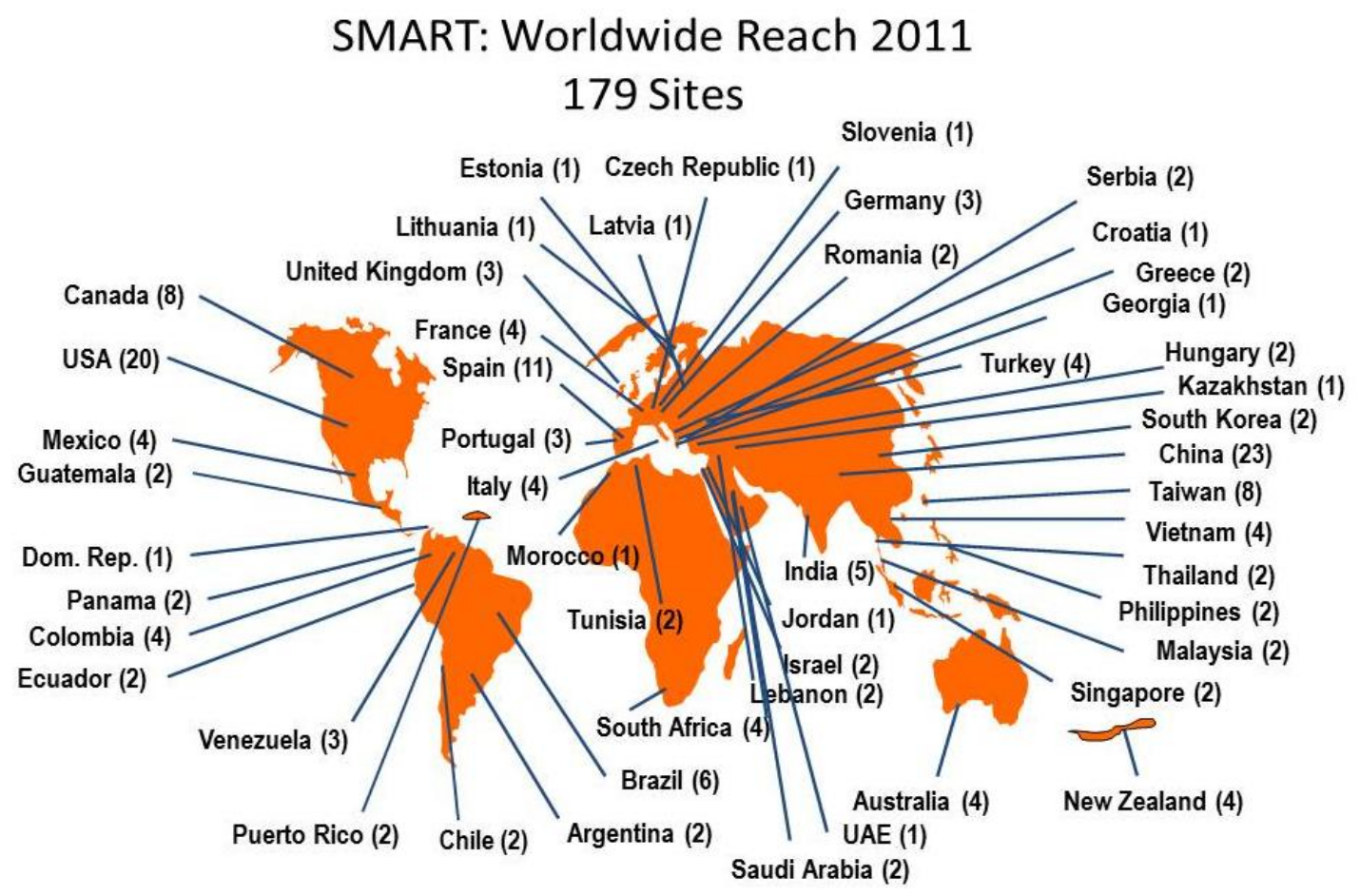

\subsection{Susceptibility Testing}

Isolates were tested for antimicrobial susceptibility using custom MicroScan dehydrated broth microdilution panels (Siemens Medical Solutions Diagnostics, West Sacramento, CA, USA) at the central laboratory. Minimum inhibitory concentrations (MICs), Clinical and Laboratory Standards Institute (CLSI) guidelines were utilized for testing [3]. The following antimicrobial agents were included on the panels with their dilution ranges (expressed in $\mathrm{mg} / \mathrm{L}$ ):

- ertapenem 0.03-4,

- imipenem 0.06-8,

- cefepime $0.5-32$,

- ceftazidime 0.5-128,

- ceftazidime-clavulanic acid 0.12/4-16/4,

- cefoxitin 2-16,

- ciprofloxacin 0.25-2,

- amikacin 4-32,

- levofloxacin 0.5-4,

- cefotaxime $0.5-128$,

- cefotaxime-clavulanic acid 0.12/4-16/4,

- piperacillin-tazobactam 2/4-64/4,

- ampicillin-sulbactam 2/2-16/2, and

- ceftriaxone 1-32.

MIC interpretive criteria of the most recent CLSI documents were followed for all pathogens and antimicrobial agents [4]. 
Using CLSI guidelines, Eschericia coli, Klebsiella pneumoniae, K. oxytoca, and Proteus mirabilis were classified as ESBL producers if there was at least an eight-fold reduction (i.e., three doubling dilutions) of the MIC for ceftazidime or cefotaxime tested in combination with clavulanic acid versus their MIC values when tested alone [4]. Trends were assessed for statistical significance using the Cochrane-Armitage test.

\subsection{Quality Control}

Quality control testing (QC) was performed each day of testing using the CLSI recommended American Type Culture Collection (ATCC) QC strains: E. coli ATCC 25922, E. coli ATCC 35218, Pseudomonas aeruginosa ATCC 27853, and K. pneumoniae ATCC 700603 (positive ESBL control). Results were included in the analysis only when corresponding QC isolates tested within the acceptable range according to CLSI guidelines [4].

\section{Results and Discussion}

Over the course of the SMART study, the five most commonly isolated Gram-negative pathogens from IAIs were E. coli, K. pneumoniae, P. aeruginosa. Enterobacter cloacae and P. mirabilis (Table 1). This was very similar across all geographical regions, although Acinetobacter baumannii was more prevalent than P. mirabilis in Asia (4.6\% vs. 2.1\%) and K. oxytoca more prevalent than P. mirabilis in the South Pacific (3.8\% vs. $3.0 \%$, data not shown). The same species were also the most frequent pathogens associated with UTIs (Table 2) with no difference between geographical regions.

Table 1. Top five most prevalent Gram-negative bacteria associated with intra-abdominal infections from SMART between 2002 and 2011.

\begin{tabular}{ccc}
\hline IAI Pathogen & $\mathbf{N}$ & $\mathbf{\%}$ \\
\hline Escherichia coli & 43,973 & 47.8 \\
Klebsiella pneumoniae & 13,385 & 14.5 \\
Pseudomonas aeruginosa & 8,674 & 9.4 \\
Enterobacter cloacae & 5,564 & 6.0 \\
Proteus mirabilis & 3,282 & 3.6 \\
Other & 17,208 & 18.7 \\
\hline Total & 92,086 & 100.0 \\
\hline
\end{tabular}

Table 2. Top five most prevalent Gram-negative bacteria associated with urinary-tract infections from SMART between 2009 and 2011.

\begin{tabular}{ccc}
\hline UTI Pathogen & $\mathbf{N}$ & $\mathbf{\%}$ \\
\hline Escherichia coli & 10,956 & 44.3 \\
Klebsiella pneumoniae & 2,906 & 11.8 \\
Pseudomonas aeruginosa & 1,331 & 5.4 \\
Proteus mirabilis & 1,137 & 4.6 \\
Enterobacter cloacae & 617 & 2.5 \\
Other & 7,758 & 31.4 \\
\hline Total & 24,705 & 100.0 \\
\hline
\end{tabular}


One particular cause for concern is the prevalence of ESBLs in the clinical setting. In the SMART study, all E. coli, K. pneumoniae, K. oxytoca and P. mirabilis were phenotypically characterised for the presence of ESBL and carbapenemase enzymes. The prevalence of ESBLs in IAIs in the SMART study by region is shown in Figures 2 and 3. It can be seen that ESBL rates are particularly high in Asia, Latin America and the Middle East, which has been noted elsewhere [5]. The prevalence of ESBLs intra-abdominal infections has steadily increased over time for in Asia. Europe, Latin America, Middle East (all $p$-values < 0.0001), North America $(p$-value $=0.03$ ) and South Pacific ( $p$-value $=0.009$ ). Interestingly the trend for ESBls in intra-abdominal infection isolates from Africa is a decreased over time, which is statistically significant ( $p$-value $=0.037$ ). For UTI pathogens the changing prevalence of ESBLs is less dramatic with only significant increases found in the Middle East $(p$-value $<0.0001)$ and Asia $(p$-value $=0.0002)$.

Figure 2. Prevalence of ESBLs in E. coli, K. pneumonia, K. oxytoca and P. mirabilis from intra-abdominal infections by region from SMART 2002 to 2011.

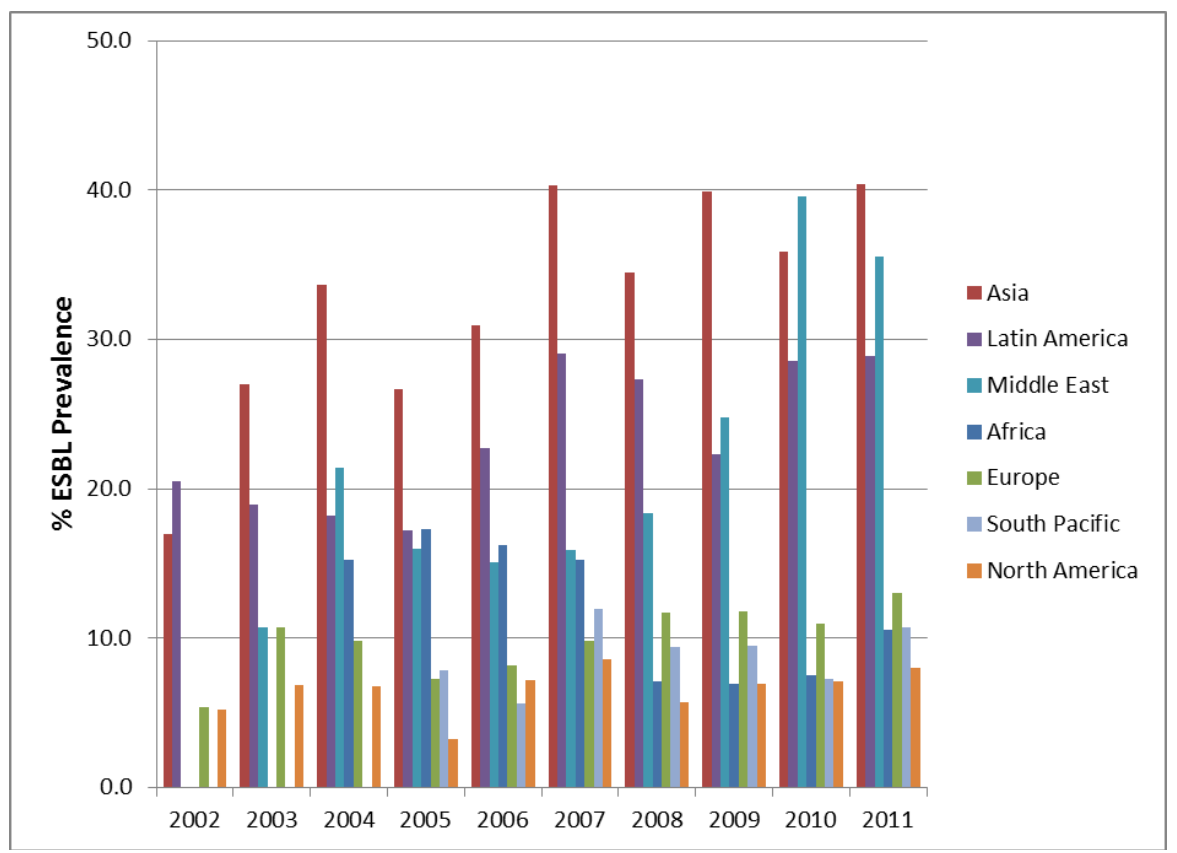

Figure 3. Prevalence of ESBLs in E. coli, K. pneumonia, K. oxytoca and P. mirabilis from urinary-tract infections by region from SMART 2009 to 2011.

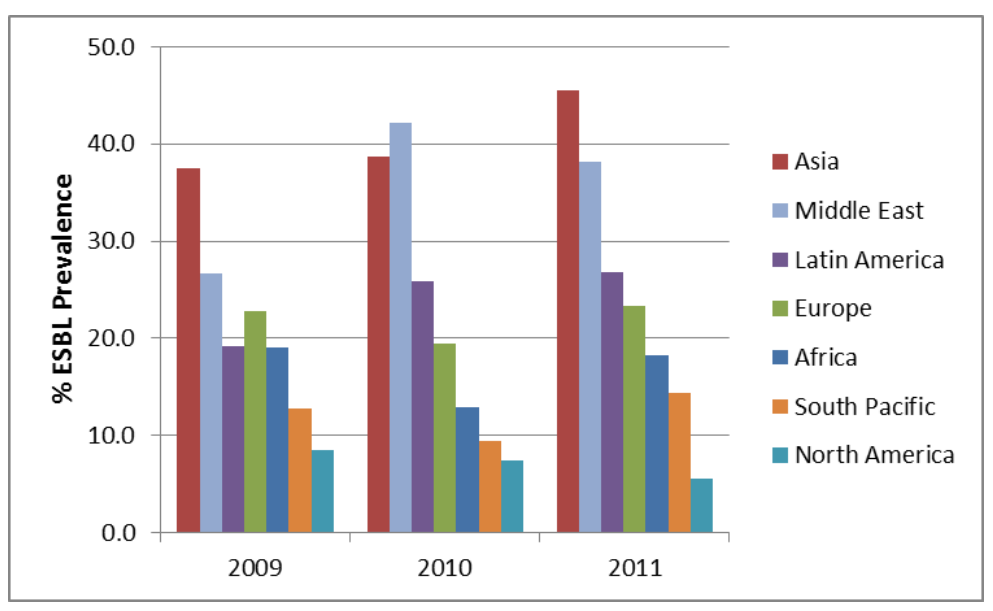


Molecular analysis of ESBLs and also carbapenemases is an important component of the SMART study and details of the methodology employed are described by Lascols et al. [6]. A recent analysis of ertapenem-resistant isolates from SMART indicate that a range of carbapenemase genes, associated with numerous ESBLs and/or AmpC backgrounds, were found among Enterobacteriaceae isolated during the study. Many of these ertapenem non-susceptible strains were clonally related and carried various combinations of $\beta$-lactamases [7]. The most common carbapenemase found in isolates collected in Indian hospitals was NDM-1 [7], despite the fact that this carbapenemase was only discovered fairly recently [8]. This carbapenemase was found on a diverse collection of Enterobacteriaceae [7], as describe by others previously [9-11]. A separate analysis of isolates from the Asia-Pacific region found that carbapenemases were almost exclusively detected among the Enterobacteriaceae isolates collected from India, with some IMP $\beta$-lactamases also detected in the Philippines and Australia [12]. In this study, K. pneumoniae had a slightly lower higher resistance rate to imipenem compared to E. coli (Figures 6-9). The main carbapenem resistance mechanisms involve a KPC enzyme, usually in combination with an SHV [7]. Surveillance studies, such as the SMART program, process a large number of bacteria providing the opportunity to identify and track novel resistance mechanisms. Our extensive molecular analysis has identified a new bla $a_{\mathrm{SHV}}$ variant, bla $_{\mathrm{SHV}-129}$, and a new bla $a_{\mathrm{KPC}}$ variant, bla $a_{\mathrm{KPC}-11}$, as well as confirming the dominance of certain ESBL types, i.e., CTX-M-15 [6]. Surveillance studies are important in detecting changes in resistance and epidemiology as well as the future evolution of bacterial resistance.

E. coli isolates from both IAIs and UTIs demonstrate consistently low resistance to carbapenems (as demonstrated by imipenem in Figures 4-6) since the beginning of SMART. Other antimicrobial agents are less active, especially against ESBL-positive E. coli.

Figure 4. Susceptibility of $E$. coli from intra-abdominal infections to imipenem (IMI-S) and prevalence of ESBLs by region from SMART 2002 to 2011.

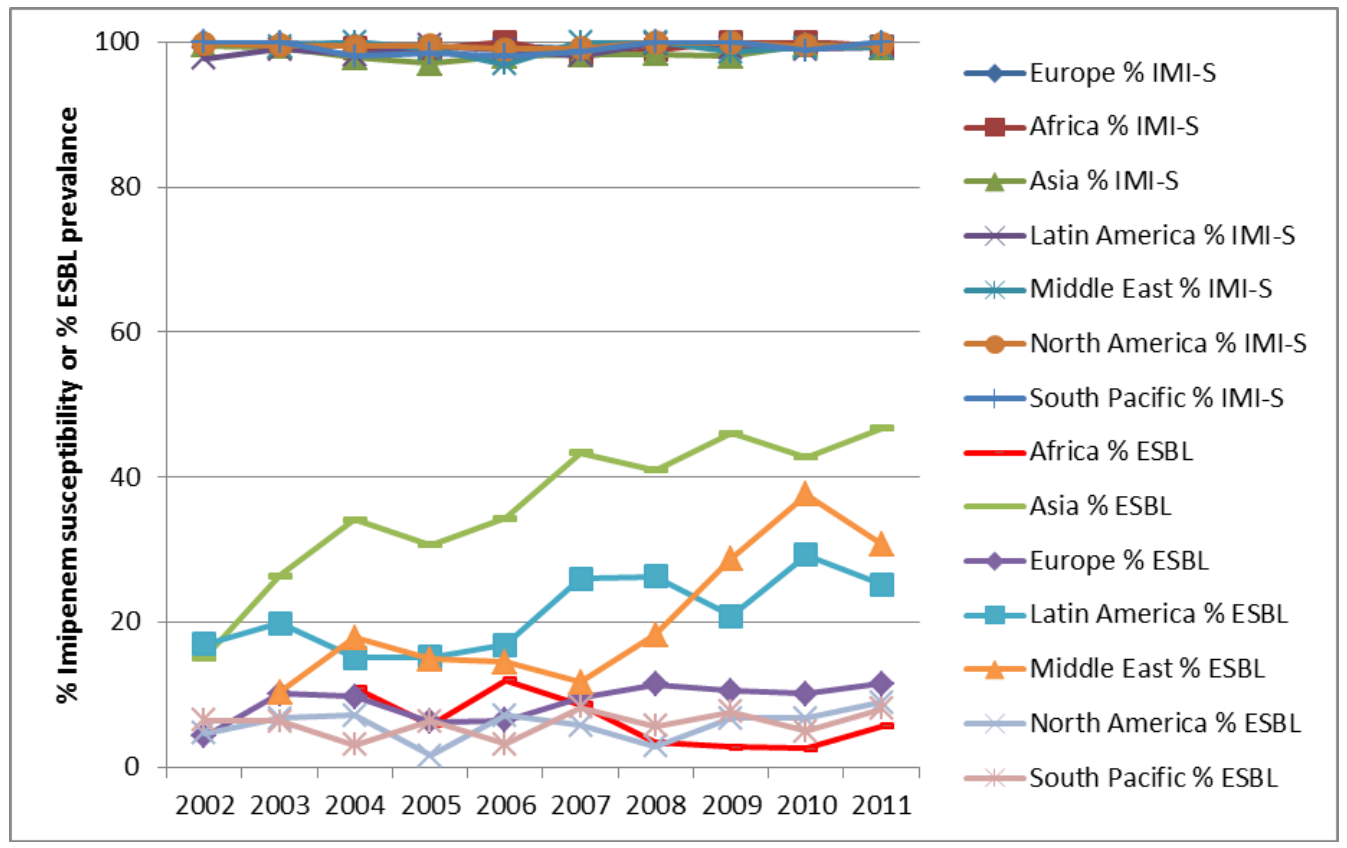


Figure 5. Susceptibility of $K$. pneumoniae from intra-abdominal infections to imipenem (IMI-S) and prevalence of ESBLs by region from SMART 2002 to 2011.

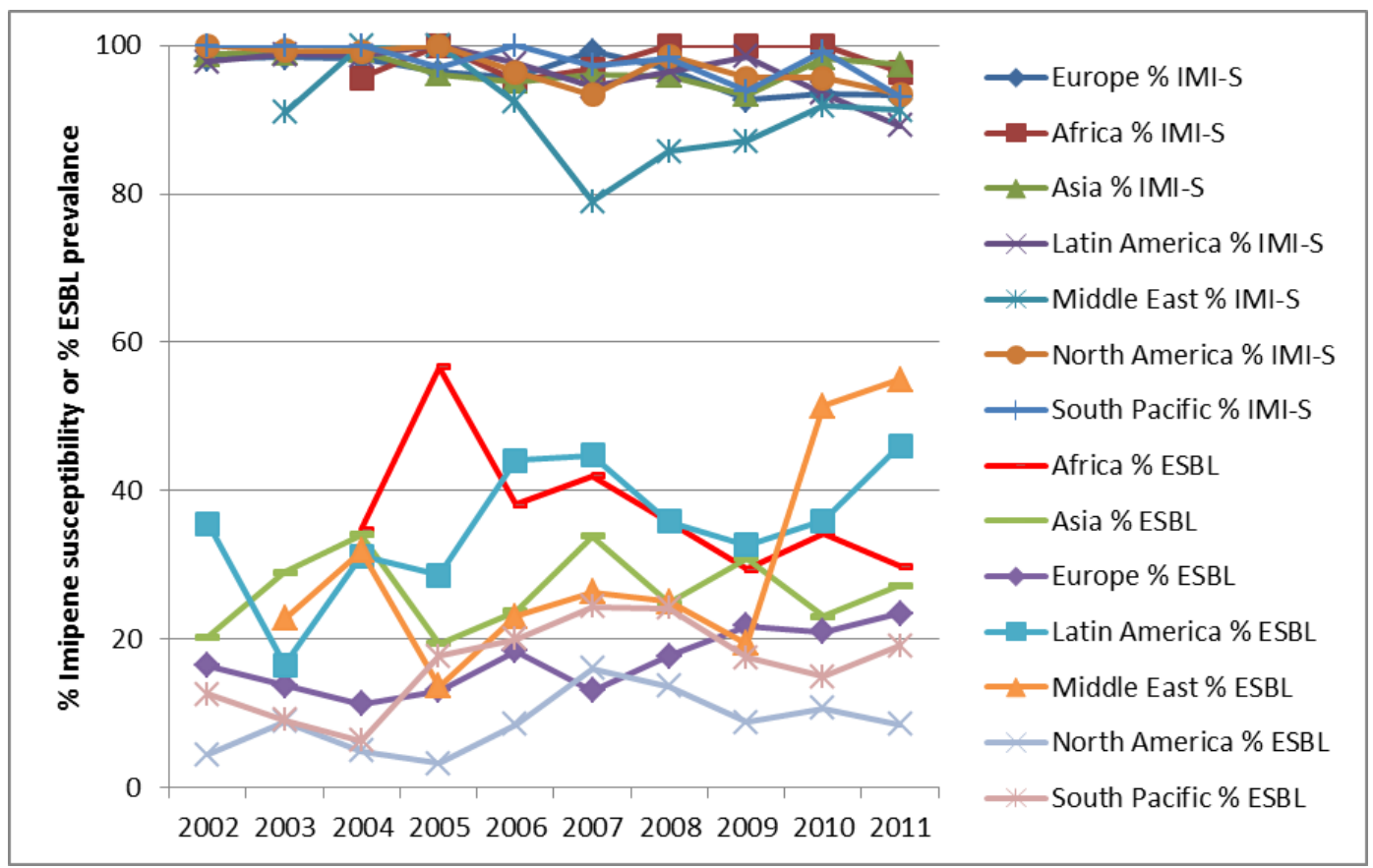

Figure 6. Susceptibility of E. coli from urinary-tract infections to imipenem (IMI-S) and prevalence of ESBLs by region from SMART 2009 to 2011.

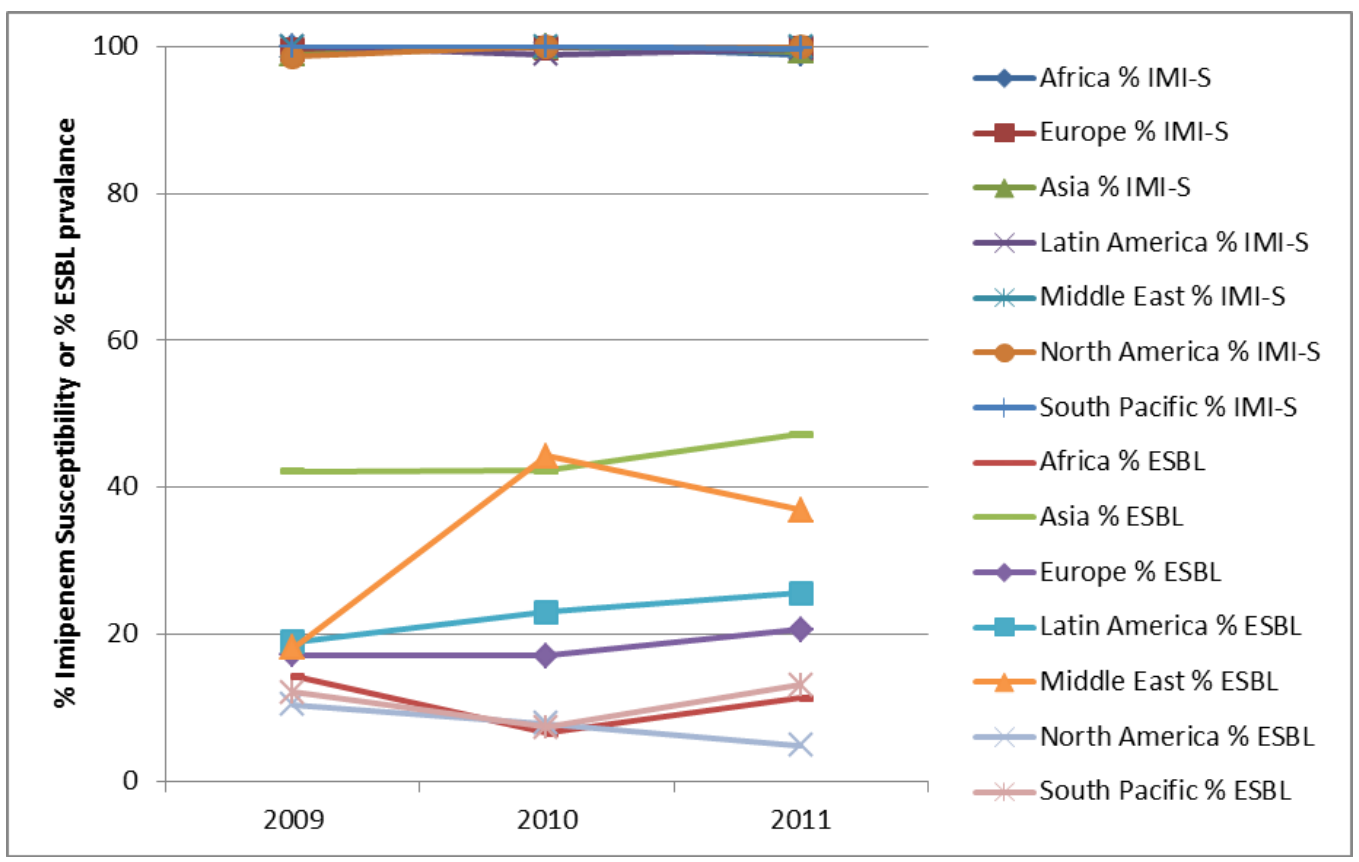

In addition to the expected increased resistance to $\beta$-lactams, fluoroquinolone resistance in ESBL-positive E. coli causing intra-abdominal infections ranges from $60 \%$ to $93 \%$ in India, China, North America, Europe and South Africa [7,13-18]. Furthermore, the vast majority of Gram-negative pathogens causing UTIs are resistant to fluoroquinolones [19,20]. For all antimicrobial agents, apart from carbapenems, it has been observed that E. coli from hospital-associated infections are less susceptible compared to community-associated infections [21]. 
$K$. pneumoniae also continue to remain susceptibile to carbapenems, especially those from UTIs (Figure 7). Although carbapenem activity against isolates from IAIs is also high (Figure 5), it is slightly lower than activity against $K$. pneumoniae isolates from UTIs or E. coli. Carbapenem activity was highest among the tested antimicrobial agents as recently demonstrated against $K$. pneumoniae from China where cephalosporins, ampicillin-sulbactam, and fluoroquinolones have 40\%-50\% resistance [13], presumably related to the high ESBL rates in this region. In other parts of the world, ESBL-positive $K$. pneumoniae are highly resistant to fluoroquinolones and $\beta$-lactams [14-16,21], as described with E. coli. Also, as with E. coli, K. pneumoniae causing hospital infections are more resistant to antimicrobial agents than those that are community-associated [21].

Figure 7. Susceptibility of $K$. pneumoniae from urinary-tract infections to imipenem (IMI-S) and prevalence of ESBLs by region from SMART 2009 to 2011.

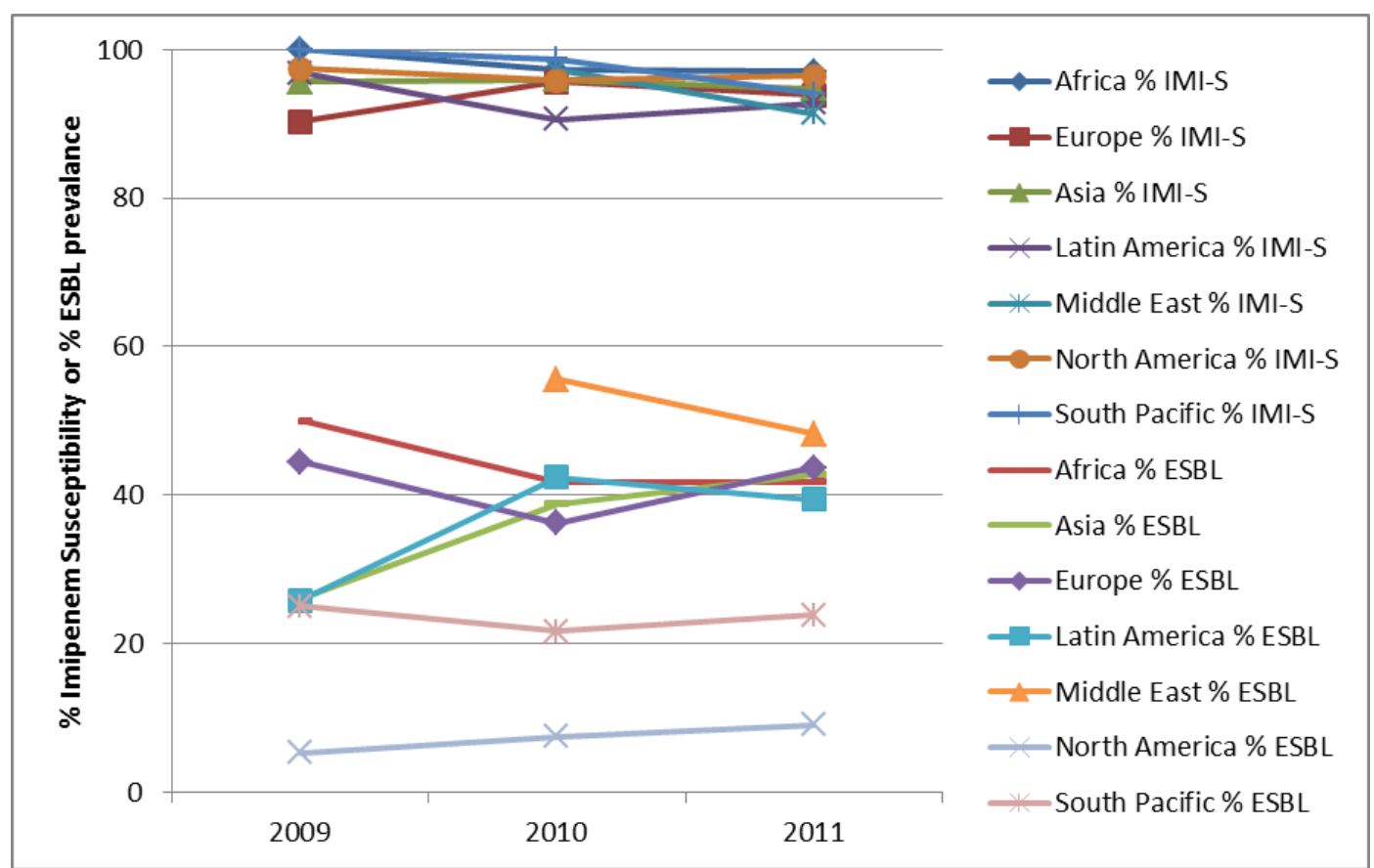

P. aeruginosa was the third most common pathogen with IAIs and UTIs at a rate of 5 and $10 \%$, respectively (Tables 1 and 2). Imipenem resistance ranged between $20 \%$ and $40 \%$ in these infection types in various regions of the world (Figures 8 and 9). Although overall lower carbapenem resistance was observed among $E$. coli and $K$. pneumoniae compared with $P$. aeruginosa, the activity of imipenem was greater against $P$. aeruginosa compared to most other classes of antimicrobial agents. The resistance of $P$. aeruginosa to fluoroquinolones has significantly increased over time in North America from approximately $22 \%$ in 2005 to $33 \%$ in 2010 while imipenem activity remained unchanged at $20 \%$ resistance. During this ten-year period, resistance to piperacillin-tazobactam, cefepime and ceftazidime remained unchanged at 23 to $26 \%$ [22]. 
Figure 8. Susceptibility of $P$. aeruginosa from intra-abdominal infections to imipenem (IMI-S) by region from SMART 2002 to 2011.

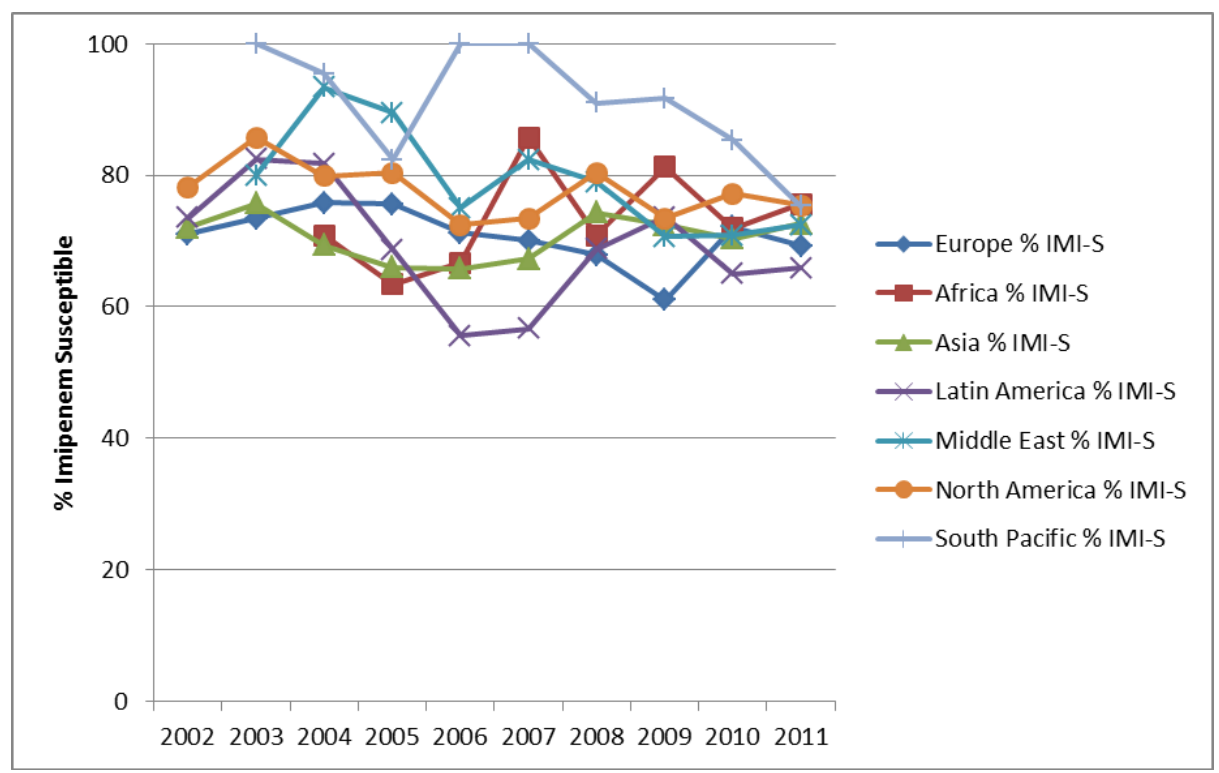

Figure 9. Susceptibility of $P$. aeruginosa from urinary-tract infections to imipenem (IMI-S) by region from SMART 2009 to 2011.

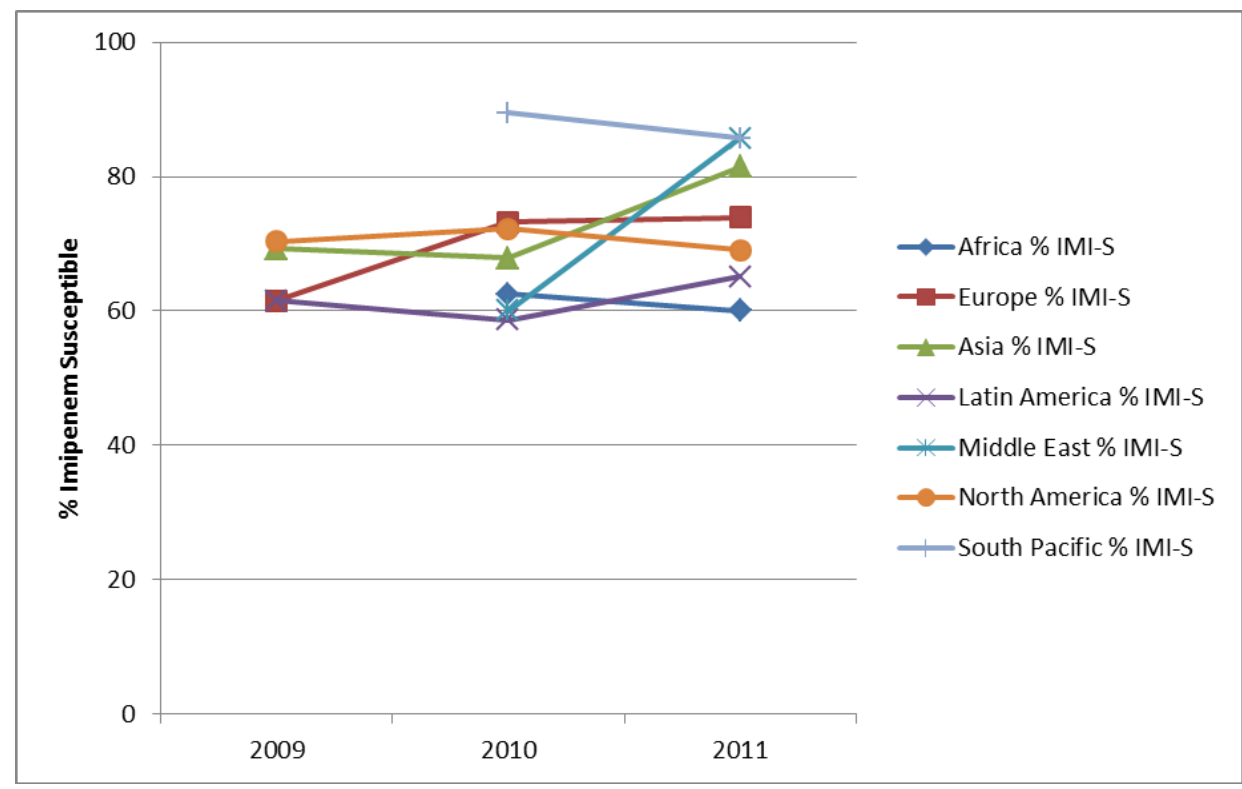

Interestingly, antibiotic resistance decreased over time for amikacin where resistance decreased from $10.6 \%$ in 2005 to $3.3 \%$ in 2010 in North America [22]. The relative activity of antimicrobials is not consistent between different regions of the world. For example, in South Africa, piperacillin-tazobactam resistance in $P$. aeruginosa was $8 \%$ but cefepime, ceftazidime and imipenem resistance was around 25 to $26 \%$. Furthermore, in contrast to the much higher rate in North America, amikacin resistance in this species in South Africa was 27\% [16]. In China (2009), the resistance of P. aeruginosa to amikacin was $12 \%$ whereas piperacillin-tazobactam was $8 \%$ [13]. Overall, all antimicrobial classes were less effective against $P$. aeruginosa hospital-associated infections compared to community-associated infections [21]. 
Clinical microbiologists and surveillance studies rely on accurate breakpoints to determine resistance rates of bacteria to antimicrobial agents based upon pharmacological and epidemiological data. These breakpoints are subject to change as new clinical or pharmacokinetic data are provided to the scientific community. CLSI and EUCAST update breakpoints annually. In 2010 CLSI reduced the breakpoints for carbapenems against Enterobacteriaceae, such that the susceptible breakpoint for ertapenem was reduced from 2 to $0.25 \mu \mathrm{g} / \mathrm{mL}$ [23]. Therefore, isolates with ertapenem MIC of 0.5 and $1 \mu \mathrm{g} / \mathrm{mL}$ were no longer considered susceptible. However, subsequent clinical data and MIC distributions and molecular analysis from the SMART study indicated that isolates with ertapenem MIC of $0.5 \mu \mathrm{g} / \mathrm{mL}$ do not contain carbapenemases and therefore should respond to ertapenem therapy [24]. Based upon these data, the CLSI susceptible breakpoints for ertapenem were increased to $0.5 \mu \mathrm{g} / \mathrm{mL}$ in 2012 [25]. Data derived from the SMART study provided essential information for determining the most appropriate epidemiological breakpoints for clinical use.

\section{Conclusions}

The SMART study has become an invaluable resource to track the resistance of Gram-negative bacteria to antimicrobial agents over time and a contemporary view of the most relative prevalence of the pathogens associated with IAIs and UTIs. Importantly, the assessment of molecular mechanisms of resistance is vital not just for epidemiological purposes but also for determining appropriate breakpoints of antimicrobial agents critical to patient care. The data produced have been able to highlight the differences in antimicrobial agent resistance between various regions of the world and these data demonstrate the continued utility of carbapenems in the treatment of these infections.

\section{Acknowedgements}

The authors thank all of the investigators and centres involved in the study. The study was funded by Merck \& Company, Inc.

\section{Conflicts of Interest}

The authors declare no conflict of interest.

\section{References}

1. Hawser, S. Surveillance programmes and antibiotic resistance: Worldwide and regional monitoring of antibiotic resistance trends. Antibiotic Resist. 2012, 211, 31-43.

2. Felmingham, D.; White, A.R.; Jacobs, M.R.; Appelbaum, P.C.; Poupard, J.; Miller, L.A.; Grüneberg, R.N. The Alexander Project: the benefits from a decade of surveillance. J. Antimicrob. Chemother. 2005, 56, ii3-ii21.

3. Methods for Dilution Antimicrobial Susceptibility Tests for Bacteria That Grow Aerobically; Approved Standards - Ninth Edition; CLSI document M07-A9; Clinical Laboratory Standards Institute: Wayne, PA, USA, 2012. 
4. Performance Standards for Antimicrobial Susceptibility Testing, Twenty-second Informational Supplement; CLSI document M100-S23; Clinical and Laboratory Standards Institute (CLSI): Wayne, PA, USA, 2013.

5. Hawser, S.P.; Bouchillon, S.K.; Hoban, D.J.; Badal, R.E. In vitro susceptibilities of aerobic and facultative anaerobic Gram-negative bacilli from patients with intra-abdominal infections worldwide from 2005-2007: Results from the SMART study. Int. J. Antimicrob. Agents. 2009, 34, 585-588.

6. $\quad$ Lascols, C.; Hackel, M.; Hujer, A.M.; Marshall, S.H.; Bouchillon, S.K.; Hoban, D.J.; Hawser, S.P.; Badal, R.E.; Bonomo, R.A. Using nucleic acid microarrays to perform molecular epidemiology and detect novel $\beta$-lactamases: A snapshot of extended-spectrum $\beta$-lactamases throughout the world. J. Clin. Microbiol. 2012, 50, 1632-1639.

7. Hawser, S.P.; Bouchillon, S.K.; Lascols, C.; Hackel, M.; Hoban, D.J.; Badal, R.E.; Woodford, N.; Livermore, D.M. Susceptibility of Klebsiella pneumoniae isolates from intra-abdominal infections and molecular characterization of ertapenem-resistant isolates. Antimicrob. Agents Chemother. 2011, 55, 3917-3921.

8. Yong, D.; Toleman, M.A.; Giske, C.G.; Cho, H.S. Sundman, K.; Lee, K.; Walsh, T.R. Characterization of a new metallo- $\beta$-lactamase gene, bla(NDM-1), and a novel erythromycin esterase gene carried on a unique genetic structure in Klebsiella pneumoniae sequence type 14 from India. Antimicrob. Agents Chemother. 2009, 53, 5046-5054.

9. Castanheira, M.; Deshpande, L.M.; Mathai, D. Early dissemination of NDM-1- and OXA-181producing Enterobacteriaceae in Indian hospitals: Report from the SENTRY Antimicrobial Surveillance Program, 2006-2007. Antimicrob. Agents Chemother. 2010, 55, 1274-1278.

10. Kumarasamy, K.K.; Toleman, M.A.; Walsh, T.R. Emergence of a new antibiotic resistance mechanism in India, Pakistan, and the UK: A molecular, biological, and epidemiological study. Lancet Infect. Dis. 2010, 10, 597-602.

11. Lascols, C.; Hackel, M.; Marshall, S.H.; Hujer, A.M.; Bouchillon, S.; Badal, R.; Hoban, D.; Bonomo, R.A. Increasing prevalence and dissemination of NDM-1 metallo- $\beta$-lactamase in India: Data from the SMART study (2009). J. Antimicrob. Chemother. 2011, 66, 1992-1997.

12. Sheng, W.H.; Badal, R.E.; Hsueh, P.R.; SMART Program. Distribution of extended-spectrum $\beta$-lactamases, AmpC $\beta$-lactamases, and carbapenemases among Enterobacteriaceae isolates causing intra-abdominal infections in the Asia-Pacific region: Results of the study for Monitoring Antimicrobial Resistance Trends (SMART). Antimicrob. Agents Chemother. 2013, 57, 2981-2988.

13. Yang, Q.; Wang, H.; Chen, M.; Ni, Y.; Yu, Y.; Hu, B.; Sun, Z.; Huang, W.; Hu, Y.; Ye, H.; et al. Surveillance of antimicrobial susceptibility of aerobic and facultative Gram-negative bacilli isolated from patients with intra-abdominal infections in China: the 2002-2009 Study for Monitoring Antimicrobial Resistance Trends (SMART). Int. J. Antimicrob. Agents 2010, 36, 507-512.

14. Hawser, S.P.; Badal, R.E.; Bouchillon, S.K.; Hoban, D.J.; SMART India Working Group. Antibiotic susceptibility of intra-abdominal infection isolates from Indian hospitals during 2008. J. Med. Microbiol. 2010, 59, 1050-1054.

15. Hawser, S.P.; Badal, R.E.; Bouchillon, S.K.; Hoban, D.J. Trending eight years of in vitro activity of ertapenem and comparators against Escherichia coli from intra-abdominal infections in North America-SMART 2002-2009. J. Chemother. 2011, 23, 266-272. 
16. Brink, A.J.; Botha, R.F.; Poswa, X.; Senekal, M.; Badal, R.E.; Grolman, D.C.; Richards, G.A.; Feldman, C.; Boffard, K.D. Veller, M.; et al. Antimicrobial susceptibility of gram-negative pathogens isolated from patients with complicated intra-abdominal infections in South African hospitals (SMART Study 2004-2009): Impact of the new carbapenem breakpoints. Surg. Infect. 2012, 13, 43-49.

17. Yang, Q.; Zhang, H.; Wang, Y.; Xu, Y.; Chen, M.; Badal, R.E.; Wang, H.; Ni, Y.; Yu, Y.; $\mathrm{Hu}, \mathrm{B}$; et al. A ten-year surveillance for antimicrobial susceptibility of Escherichia coli and Klebsiella pneumoniae in community- and hospital-associated intra-abdominal infections in China. J. Med. Microbiol. 2013, 62, 1343-1349.

18. Hawser, S.P.; Badal, R.E.; Bouchillon, S.K.; Hoban, D.J.; Biedenbach, D.J.; Cantón, R. Paterson, D.L. Monitoring the global in vitro activity of ertapenem against Escherichia coli from intra-abdominal infections: SMART 2002-2010. Int. J. Antimicrob. Agents 2013, 41, 224-228.

19. Hoban, D.J.; Lascols, C.; Nicolle, L.E.; Badal, R.; Bouchillon, S.; Hackel, M.; Hawser, S. Antimicrobial susceptibility of Enterobacteriaceae, including molecular characterization of extended-spectrum $\beta$-lactamase-producing species, in urinary tract isolates from hospitalized patients in North America and Europe: Results from the SMART study 2009-2010. Diagn. Microbiol. Infect. Dis. 2012, 74, 62-67.

20. Bouchillon, S.K.; Badal, R.E.; Hoban, D.J.; Hawser, S.P. Antimicrobial Susceptibility of Inpatient Urinary Tract Isolates of Gram-Negative Bacilli in the United States: Results from the Study for Monitoring Antimicrobial Resistance Trends (SMART) Program: 2009-2011. Clin. Ther. 2013, 35, 872-877.

21. Hawser, S.P.; Bouchillon, S.K.; Hoban, D.J.; Badal, R.E. Epidemiologic trends, occurrence of extended-spectrum $\beta$-lactamase production, and performance of ertapenem and comparators in patients with intra-abdominal infections: Analysis of global trend data from 2002-2007 from the SMART study. Surg. Infect. 2010, 11, 371-378.

22. Babinchak, T.; Badal, R.; Hoban, D.; Hackel, M.; Hawser, S.; Lob, S.; Bouchillon, S. Trends in susceptibility of selected gram-negative bacilli isolated from intra-abdominal infections in North America: SMART 2005-2010. Diagn. Microbiol. Infect. Dis. 2013, 76, 379-381.

23. Performance Standards for Antimicrobial Susceptibility Testing, Twenty-second Informational Supplement; CLSI document M100-S20U; Clinical Laboratory Standards Institute: Wayne, PA, USA, 2010.

24. Badal, R.E.; Lewis, J.S., III; Motyl, M.R. Changes in the CLSI Interpretive Criteria for Enterobacteriaceae and Ertapenem. Clin. Microbiol. Newsl. 2011, 33, 185-186.

25. Performance Standards for Antimicrobial Susceptibility Testing, Twenty-second Informational Supplement; CLSI document M100-S22; Clinical and Laboratory Standards Institute (CLSI): Wayne, PA, USA, 2012.

(C) 2013 by the authors; licensee MDPI, Basel, Switzerland. This article is an open access article distributed under the terms and conditions of the Creative Commons Attribution license (http://creativecommons.org/licenses/by/3.0/). 